\title{
Supervisors' perceptions regarding the Zambian HIV nurse practitioner program and integrating graduates into the Zambian health system: A descriptive cross-sectional survey
}

\author{
Debra McCullock ${ }^{* 1}$, Dorothy Chanda ${ }^{2}$, Esther Masebe ${ }^{3}$, Alexandrina Mwale ${ }^{3}$, Ruth Wahila ${ }^{2}$, Lynda Wilson ${ }^{1}$ \\ ${ }^{1}$ University of Alabama at Birmingham, United States \\ ${ }^{2}$ Department of Nursing Sciences, School of Medicine, University of Zambia, Zambia \\ ${ }^{3}$ Lusaka School of Nursing and Midwifery, Lusaka, Zambia
}

Received: December 21, 2015

DOI: $10.5430 /$ jnep.v6n7p140
Accepted: February 15, $2016 \quad$ Online Published: April 13, 2016

URL: http://dx.doi.org/10.5430/jnep.v6n7p140

\begin{abstract}
Introduction: The disease burden of HIV and AIDS in Zambia is among the highest in the world. In 2009 the HIV Nurse Practitioner (HNP) program was implemented to address the disease burden of HIV and AIDS that is worsened by a critical shortage of health care workers and in Zambia. The objective of this study was to analyze the perceptions of supervisors of the first three cohorts of graduates of the HNP program regarding the HNP role, the impact of the role on the quality of care for HIV patients and their families, and perceived challenges in integrating the HNP graduates into the Zambian health system.

Methods: This paper reports findings from a cross-sectional survey of the supervisors from the first three cohorts of Zambian HNP graduates who completed the program between 2010-2012. Thematic analysis was used to identify themes from the responses of 60 supervisors. The project received approval from the University of Zambia Biomedical Research Ethics Committee and from the University of Alabama at Birmingham Institutional Review Board.

Results: The HNP graduates' supervisors reported an understanding of the role, and a positive perception of the impact of the HNP cadre on the quality of HIV and AIDS care for patients and their families. The perceived challenges to integration of the HNP into the Zambian health system included the need to clarify job descriptions and responsibilities for the new role within the Ministry of Health, addressing issues related to the shortage of nurses to meet other health needs, ensuring appropriate referral of complex cases, and providing ongoing HNP supervision.

Conclusion: The findings in this study can be used to guide the development of this program and the development of future task-shifting programs to provide comprehensive care to patients with HIV and AIDS.
\end{abstract}

Key Words: Nurse, Practitioner, Scope, Task shifting, Task sharing, HIV, AIDS, Zambia

\section{INTRODUCTION}

Sub-Saharan African countries are faced with an estimated shortage of 2.4 million physicians and nurses. ${ }^{[1,2]}$ This short- age creates challenges in accessing preventive, routine, and disease-related healthcare services and is a significant barrier to achieving the United Nations Millennium Development

\footnotetext{
*Correspondence: Debra McCullock, DNP, MS, APRN-CNP, RN-BC; Email: debmccullock@gmail.com; Address: University of Alabama at Birmingham School of Nursing, United States.
} 
Goal \#6: Reversing the Spread of HIV and AIDS, Malaria and other major diseases, ${ }^{[3]}$ and the 2015 Sustainable Development Goal \#3: Ensure healthy lives and promote wellbeing for all at all ages. ${ }^{[4]}$ Achievement of these goals would make significant contributions in the fight against HIV/AIDS.

Zambia is a landlocked, sub-Saharan, developing nation of 14.6 million people in southern central Africa with a median age of 16.7 years and a life expectancy of 51.8 years. ${ }^{[5]}$ Zambia was identified as one of the 49 Least Developed Countries (LDC) by the United Nations. ${ }^{[6]}$ Sixty-eight percent of the population is below the International Poverty Line, living on less than $\$ 1.25$ per day. Zambia has the fifth highest percentage of the population living at this level of poverty globally among $\mathrm{LDCs}^{[6]}$ and is challenged with a workforce of 8.5 skilled health care workers / 10,000 population ${ }^{[2]}$ and 0.7 physicians per 1,000 population. ${ }^{[5]}$ This compares to 122.3 skilled health workers / 10,000 population and 2.4 physicians per 1,000 population in the United States. ${ }^{[5]}$ Extreme, persistent, and widespread poverty complicates the shortage of healthcare workers addressing the disease burden of HIV and AIDS.

The disease burden of HIV and AIDS in Zambia is among the highest in the world. The adult prevalence rate of HIV in Zambia is $14.3 \%,{ }^{[7]}$ seventh in the world. ${ }^{[8]}$ The HIV mortality rate in adults in Zambia is $52 \%$, and HIV is the most significant cause of adult mortality in Zambia. ${ }^{[9,10]}$ The HIV mortality rate in children under age 5 years in subSaharan Africa is 15 times more than the rate in developed nations. ${ }^{[11]}$ The World Health Organization (WHO) has indicated that scaling-up the development of effective health services will result in more than $60 \%$ of these child deaths being avoided. ${ }^{[12]}$

Since 1991, the Zambian Ministry of Health National Strategic Plan has identified the need to improve access to equitable, quality, cost-effective health services. ${ }^{[13,14]}$ In 2009, the General Nursing Council of Zambia, in partnership with the Zambian Ministry of Health, the University of Zambia and other Zambian and international partners, initiated the HIV Nurse Practitioner (HNP) program in response to the critical shortage of health care providers in Zambia. The Zambian Nurses and Midwives Practice Act of 1997 specifies that Zambian nurses are allowed to prescribe selected medications if they have completed an approved educational program. ${ }^{[15]}$

The HNP program curriculum was approved by the Zambian Ministry of Health and by the Zambian General Nursing Council, and graduates of the program were authorized to provide first-level care (including initiation of antiretroviral therapy) to patients with HIV and AIDS. It was anticipated that providing advanced, specialized academic training and clinical mentorship for nurses would reduce delays in treatment and improve quality of care for patients with HIV and AIDS. The primary focus of this training program was to meet the urgent need for front-line, primary HIV care services in Zambia. ${ }^{[16]}$

A total of 92 nurses completed the 1-year program during its first 3 years. As of September 2015 a total of 169 students have completed the program, and 33 were currently enrolled and still in training. Both Enrolled (two-year) and Registered (three-year) trained nurses are permitted to enter the program. The program consists of didactic classwork and extensive clinical mentoring. The program structure is illustrated in Figure $1 .{ }^{[16]}$

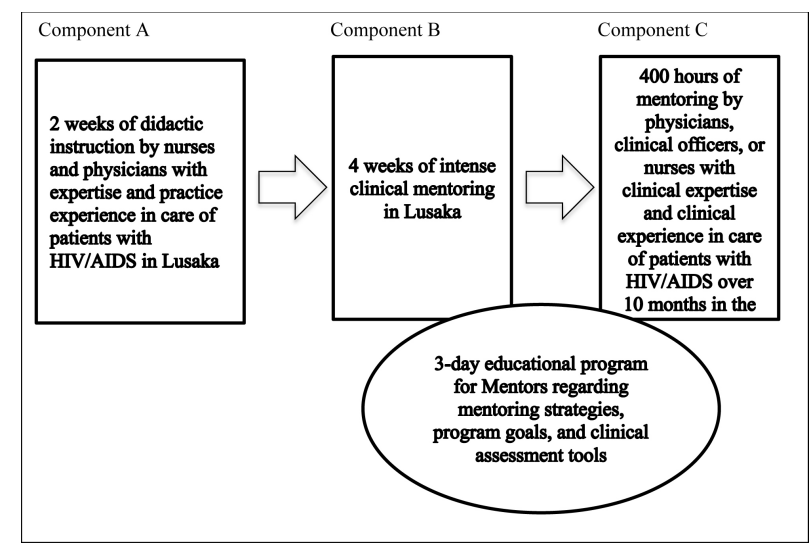

Figure 1. Components of the HNP Program

Two papers have been previously published describing the development and implementation of the Zambian HNP program and outcomes from the first two cohorts of graduates. ${ }^{[16,17]}$ This paper presents findings from surveys that were completed by 60 supervisors of the 92 graduates from the first three cohorts of the program related to their perceptions of the role of the HNP, potential contributions and challenges associated with integration of this cadre of health worker into the Zambian health system, and recommendations for changes to the HNP program. The questions that guided the study were:

(1) What were supervisors' perceptions of the role of the HNP graduate?

(2) What were supervisors' perceptions of ways in which the HNP role would affect care of patients with HIV in Zambia?

(3) What were supervisors' perceptions of challenges to the integration of the HNP role into their clinical sites?

(4) What were supervisors' perceptions of challenges to the integration of the HNP role into the general Zambian health system? 
(5) What were supervisors' recommendations about strategies to improve the HNP program?

\section{Literature review}

In 2008, the WHO published 22 global recommendations for the implementation of task-shifting as a strategy to rapidly increase access to HIV care services. Recommendations \#18 and \#19 speak to the nurse's role and the potential impact on the system of care by making more efficient use of the available human resource cadre of nurses and training them with the knowledge to safely assume tasks traditionally administered by the physician. ${ }^{[18]}$ Significant evidence has been published in support of shifting designated tasks from the physician to a trained nurse as a high-quality, highly accepted, and cost-effective strategy to address the shortage of human resource toward scaling-up services in critical shortage areas. ${ }^{[19-21]}$

Five papers were identified that presented reviews of existing studies about the effectiveness, acceptability, or costeffectiveness of task-shifting to nurses. Callaghan, Ford and Scheider conducted a systematic review of 84 published articles related to task-shifting for HIV treatment in Africa. ${ }^{[22]}$ This review included task-shifting to nurses as well as other health worker cadres. These authors concluded that taskshifting substantially increases access to HIV care in underserved areas, and should be considered when human resource shortages impede program development. The authors reported that task-shifting created more time for physicians to attend to more complicated cases and also reduced bottlenecks in clinics by improving efficiency. Emdin and colleagues conducted a meta-analysis search of nurses and clinical officers providing HIV care. Internet searches related to three themes was performed: HIV/AIDS, ART and task shifting. Of the 2,021 studies identified, 9 were eligible for the meta-analysis and 3 were only eligible for the qualitative review. All of these studies were conducted in Africa and included a total of 59,666 participants. The authors concluded that outcomes of HIV treatment by non-physicians and physicians were the same, and that patients perceived nurses as providing more holistic care, resulting in greater levels of patient satisfaction. ${ }^{[23]}$ Iwu and Holzemer reviewed 11 studies related to outcomes of task-shifting to nurses. The authors concluded that the overall impact of task-shifting to nurses was positive, and the clinical outcomes were comparable to physician managed patient outcomes. ${ }^{[24]}$ Kredo and colleagues reviewed 10 studies regarding task-shifting HIV care in Africa from doctors to nurses and clinical of ficers. They reported moderate quality evidence that task shifting does not decrease the quality of care, and that patients lost to follow-up were decreased in nurse-initiated care. ${ }^{[25]}$ Mdege, Chindove and Ali conducted a systematic review of 6 published studies including 19,767 patients evaluating task-shifting models against other interventions based on outcomes including adherence to ART medications, clinic follow-up visits, costs and effectiveness. The findings suggested that task-shifting can potentially reduce cost and be effective in providing access to ART medications without compromising health outcomes for patients. ${ }^{[33]}$

Nine papers were identified that reported individual studies evaluating outcomes of task shifting to nurses. Bemelmans and colleagues studied a district-wide task-shifting model in Malawi, in which antiretroviral therapy (ART) initiation responsibilities were assigned to non-physicians. Findings indicated an increased access to ART, despite ongoing staff shortages. ART enrollment was doubled after shifting the responsibility for ART initiations. ${ }^{[26]}$ Boulle and colleagues studied data from 459 patients over a 2-year period from the Stratall trial in rural Cameroon comparing patient outcomes from nurse and physician visits. Findings indicated that task-shifting to nurses did not compromise patient outcomes. The authors suggested that task-shifting and investment in nursing resources for management of non-complicated patients would reduce waiting lists, reduce costs, and free up physicians time for other essential activities. ${ }^{[27]}$ Grimsrud and colleagues studied outcomes at a nurse-managed clinical site in Cape Town, South Africa. Forty-one percent of the 5,746 patients who were initiated on ART over 5 years were referred to the nurse-managed clinic when stable. The nurse clinic outcomes included lower mortality rates, and loss-to-follow-up. Virologic failure rates were similar among patients from the nurse and physician-managed clinics, and there was no increased risk for morality or virologic failure in the nurse-managed clinics. ${ }^{[28]}$ Dambisya and Matinhure conducted 34 interviews and eight focus group discussions with participants from various levels of the health system in Uganda. Respondents included policy makers, human resource managers, health workers, health worker leaders and managers, and student nurses. Respondents reported widespread task-shifting in response to the human resource shortage. The findings from this study suggested that tasks were officially shifted to trained staff and also unofficially shifted to staff with less training in response to the need, and that task-shifting was highly accepted in HIV and AIDS service delivery. ${ }^{[2]}$ Morris and colleagues implemented a task-shifting program in Zambia in which selected responsibilities were shifted from physician to clinical officer, from clinical officer to nurse, and from nurse to community health worker. Training for the task-shifting program included didactic sessions, skills training, intense mentoring, and quarterly assessments. The authors concluded that task-shifting 
was an effective strategy to scale-up staffing to address program needs. ${ }^{[30]}$ O'Malley and colleagues evaluated a pilot program in Namibia that trained 11 nurses from nine different clinical sites in nurse initiated ART management. The purpose of this study was to assess the acceptability and feasibility of task-shifting to inform policy in the context of the Namibian health system. Six-months after training was completed, researchers conducted 70 face-to-face interviews of patients, managers, nurses and physicians in nine clinics where the task-shifting intervention occurred. The findings indicated patients, nurses, physicians and healthcare workers perceived that task-shifting resulted in improvement in nursing skills, clinic flow, and patient quality of and access to care. ${ }^{[31]}$ In a study evaluating two-year outcomes of a nurseinitiated and managed HIV program in Lesotho, Cohen and colleagues observed favorable patient outcomes including feasibility and effectiveness of the nurse led initiative, improved adherence to ART, and a strengthened primary care system by task-shifting critical duties to qualified nurses. ${ }^{[32]}$ Asfaw and colleagues studied patient satisfaction levels in a task-shifting service delivery setting in four regions of Ethiopia through ART clinic exit interviews of 665 patients. Most patients (528 of the 665 patients surveyed) reported satisfaction with the services they received in the clinic, and patients who received services from nurses or health officers were significantly more likely to report satisfaction that those that were seen by doctors. ${ }^{[34]}$ Foster and McIntyre interviewed 230 patients at six facilities in the Western Cape of South Africa. ${ }^{[35]}$ Their model compared dispensing ART by an indirectly supervised pharmacist's assistant to dispensing by a trained nurse. Findings suggested that dispensing by the pharmacist's assistant is the least expensive strategy, however patients reported that they preferred receiving their anti-retroviral medications from the nurse because of convenience and fear of HIV status disclosure (two known barriers to adherence).

In summary, although a number of studies have identified positive outcomes of task-shifting in many areas of Africa, there have been few studies examining the perceptions of supervisors and other co-workers on the impact of task-shifting on the health system. Findings from the present study contribute to the existing literature by describing the perceptions of a group of supervisors of HNP students about the benefits as well as challenges associated with integration of the HNPs into the Zambian health system. The feedback regarding graduates from three successive cohorts of the HNP program offers the opportunity for a longitudinal observation of the process of training the HNP students for useful application to this program, and future programs for expansion of the nursing role, and defining the new scope of practice to effectively

Published by Sciedu Press address the shortage of health care workers in response to a health crisis such as HIV and AIDS.

\section{METHODS}

This was a cross-sectional survey of the supervisors from the first three cohorts of Zambian HNP graduates who completed the program between 2010-2012. Thematic analysis was used to identify themes from the responses of 60 supervisors. The original project received approval from the University of Zambia Biomedical Research Ethics Committee and from the University Alabama at Birmingham Institutional Review Board. The HNP Monitoring and Evaluation Team developed the survey instrument. The instrument has face validity, but there was no formal assessment of reliability.

The 92 graduates from the first 3 cohorts of the program were asked to give the survey to their workplace supervisors at the end of the 1-year HNP program (30 graduates from Year 1, 34 graduates from Year 2, and 28 graduates from Year 3). The project's Monitoring and Evaluation Team members determined that asking students to deliver the surveys to their supervisors would be a more reliable way to ensure that the supervisors received the surveys than sending them in the mail. Supervisors were asked to post mail their anonymous responses to the project coordinator when completed. The survey consisted of one question asking for information on the respondent's position within the Zambian health system, and five open-ended questions regarding the respondent's perceptions about the HNP role and the program. Two of the co-authors, the HNP Program Coordinator, and HIV Nurse Specialist (Mwale and Wahila), transcribed the supervisors' comments and entered them into an Excel database. Two of the other co-authors (McCullock and Wilson) exported the comments as RTF files into the MaxQDA-11(C) qualitative analysis program and used thematic analysis to independently code the comments to identify common themes for each survey question . After initial independent coding, these two co-authors subsequently compared their coding to achieve consensus. The coding was finally reviewed by the remaining co-authors who validated the emergent themes by consensus.

\section{Results}

Sixty supervisors, or 65\%, of the 92 total graduates' supervisors for the first three cohorts, participated in the study (25 supervisors from Year 1 graduates, 11 supervisors from Year 2 graduates, and 24 supervisors from Year 3 graduates). Supervisors were from all nine Zambian provinces. (Following this study Zambia restructured so there are now ten provinces). One supervisor described his/her role as data entry clerk, and this response was not included in the anal- 
ysis because it was not clear whether the respondent was actually a graduate's supervisor. Because the responses were anonymous, there were no data about demographic characteristics of the supervisors. Similarly, it was not possible to determine whether supervisors may have responded to the surveys collected for more than one cohort, so some of the same supervisors may have responded to more than one survey across the three years.

Findings related to each of the five study questions are presented below by describing the categories that were identified under each question, and presenting examples of comments coded in each category in italics. Table 1 illustrates the number of comments coded in each of the categories by study question.

Table 1. Frequency of Codes for Each Study Question

\begin{tabular}{|c|c|}
\hline \multicolumn{2}{|l|}{ Question 1 - Describe Your Understanding of the HNP Role } \\
\hline $\begin{array}{l}\text { 1a. Providing comprehensive care for HIV patients and families in } \\
\text { collaboration with physicians/clinicians }\end{array}$ & 100 \\
\hline 1b. Leadership and coordination of HIV services & 7 \\
\hline \multicolumn{2}{|l|}{$\begin{array}{l}\text { Question } 2 \text { - Describe Ways in which the HNP Might Affect Quality of } \\
\text { Care for HIV Patients and Families }\end{array}$} \\
\hline 2a. Positive effect on quality of care: Improving access to care & 36 \\
\hline 2b. Positive effect on quality of care: Task shifting & 15 \\
\hline 2c. Positive effect on quality of care: Improving quality of care & 55 \\
\hline 2d. Negative effect on quality of care & 1 \\
\hline \multicolumn{2}{|l|}{$\begin{array}{l}\text { Question } 3 \text { - Describe Concerns or Problems With Integrating HNP Into } \\
\text { This Clinic }\end{array}$} \\
\hline 3a. Anticipate no problems & 15 \\
\hline 3b. Anticipate positive outcomes & 16 \\
\hline 3c. Concerns with Integrating HNP Into this clinic & 34 \\
\hline \multirow{2}{*}{\multicolumn{2}{|c|}{$\begin{array}{l}\text { Question } 4 \text { - Describe Concerns or Problems with Integrating HNP Into } \\
\text { Zambian Health System }\end{array}$}} \\
\hline & \\
\hline 4a. No problems & 20 \\
\hline 4b. Anticipate positive outcomes & 8 \\
\hline 4c. Concerns about integration into Zambian health system & 38 \\
\hline \multicolumn{2}{|l|}{ Question 5 - Other Comments To Help us Improve Programs to Prepare } \\
\hline \multicolumn{2}{|l|}{ Nurses to Care for Patients with HIV/AIDS and their Families } \\
\hline 5a. Clarify HNP role and scope of practice & 6 \\
\hline 5b. Need for recognizing, compensating or motivating the HNPs & 4 \\
\hline 5c. Supportive of the program and the HNP role & 12 \\
\hline $\begin{array}{l}\text { 5d. Suggestions for selection of participants, program design, training, } \\
\text { and ongoing HNP education strategies }\end{array}$ & 22 \\
\hline 5e. Other concerns & 5 \\
\hline
\end{tabular}

\section{Question 1: Supervisors' Perceptions of the Role of the HNP Graduate}

Two codes emerged from responses to the first question asking supervisors about their perceptions of the role of the HNP:

(1a) Provision of comprehensive care in collaboration with physicians / clinicians and (1b) Leadership and coordination of HIV services.

\section{1a. Provision of Comprehensive Care.}

Comments were coded in this category if they referred to collaboration with other clinicians in caring for patients with HIV and AIDs or their families, providing care when the clinical officer ( $\mathrm{CO}$ ) or physician was not available, or providing clinical services including screening, testing, counseling, follow-up, outreach, coordination of care, managing opportunistic infections, initiating ART, caring for stable patients, or increasing access to HIV services. Examples of comments coded in this category include:

- The HIV Nurse Practitioner will be able to provide a comprehensive care to HIV patients in their health institutions by prescribing and maintaining patients on ARVs (anti-retrovirals)

- Ensure adherence to medication and follow up of patients

- Prescribing drugs and managing opportunistic infections

- Initiate ART on stable clients within the ART clinic

\section{1b. Leadership and Coordination of HIV Services.}

Comments were coded in this category if they referred to leadership, vision, coordination, or management of services for HIV patients in the absence of the $\mathrm{CO}$ or physician. Examples of comments coded in this category include:

- Liaises with community based health workers and volunteers

- To be able to run a clinic in the absence of a clinician

- The HIV Nurse Practitioner should assume leadership roles in the care and treatment of people with HIV infections

\section{Question 2: Supervisors' Perceptions of Ways in which the HNP Role Might Affect the Quality of Care Provided to Patients and Their Families}

Four themes emerged from responses to the second question asking about the affect of the HNP role on the quality of care for patients with HIV and their families. Three codes reflected positive perceptions: (2a) improving access to care, (2b) task-shifting, (2c) improving quality of care. One code reflected a negative perception on potential impact on quality of care $(2 d)$.

\section{2a. Positive Effect on Quality of Care; Improving Access to Care.}

Comments were coded in this category if they referred to improved access to care for people with HIV and their families by reducing the critical shortage in human resources, reducing waiting time for clients, reducing delays in care, and improving access to a care provider. Examples of comments coded in this category include: 
- They are able to provide a holistic care to people infected with HIV and AIDS and their families. Thereby improving services quality in under-staffed health facilities throughout the country

- Their role will positively affect the quality of care in that even in the absence of a clinician, noncomplicated cases will still be attended to. This will significantly reduce on the delays in care

\section{2b. Positive Effect on Quality of Care; Task-Shifting.}

Comments were coded in this category if they referred to task-shifting the roles of the physician, medical officer, and clinical officer to the HNP and by providing care in the absence of a clinician, freeing physicians for more complicated cases, managing clients independently and providing the same quality care as medical doctors. Examples of comments coded in this category include:

- The nurse practitioner role will affect the quality of care provided to patients by reducing the shortage in human resource, by helping medical officers or clinical officers in the task-shifting role by providing the same quality care which medical doctors would have given

- Helping medical or clinical officers in the task-shifting role

- It will improve the quality of care as they will be able to initiate, maintain ARVs, and also treat any opportunistic infections even in the absence of the clinician

- Stable patients are seen by nurse practitioners, giving more time for doctors and clinical officers to concentrate on serious cases

\section{2c. Positive Effect on Quality of Care; Improving Quality of} Care.

Comments were coded in this category if they referred to improving the quality of care by referring complicated cases in a timely manner, spending more time with patients, improving adherence, improving patient follow-up, providing care in the absence of a clinician, reducing mortality, raising the standard of management and care, improving access to counseling, providing a continuum of service, providing holistic care, improving patients' confidence, maintaining a close relationship with patients, monitoring patients more closely, involving family members to encourage patients, and supporting families of HIV patients. Examples of comments coded in this category include:

- The way in which the HIV Nurse Practitioner role affects the quality of care provided by keeping the patients taken care of, that is improving the quality of life, providing ARVs, take care of OIs (opportunistic infections) and doing lab investigations and also counseling. Also involving family members to encourage patients

- The basic training of nursing makes them to care for the patient and the family with the added training of HIV practitioner makes them complete to provide psychosocial and clinical/management of HIV in the clinic and at home

- They are able to provide a holistic care to people infected with HIV/AIDS and their families. Thereby improving services quality in understaffed health facilities throughout the country

Only one comment reflected the perception that the HNP role will have a negative impact or no impact on the quality of care of patients with HIV/AIDS:

- If the nurse is not given support during her practice by discussions or e-providers this would make her manage all patients including complex cases. This may also lead to failure in identifying when to consult or refer and consequently compromise care of patients otherwise needing specialized treatment.

\section{Question 3: Supervisors' Concerns or Perception of Potential Problems in Integrating the HNP into Their Health Care Facility.}

Three codes emerged from responses: (3a) anticipating no problems with integration of the HNP in their clinic (3b) anticipating positive outcomes of integrating the HNP into their clinic and (3c) concerns with integrating the HNP into their clinic.

3a. Anticipating No Problems with Integration of the HNP in their Clinic.

Comments were coded in this category if they indicated that there have been no problems, and/or anticipation of no problems for staff or patients with integration of the HNP into their clinic. Examples of comments coded in this category include:

- There are no concerns or problems as nurse practitioners have adequate clinical skills (and) offer their training

- In this clinic, no concerns as everyone working here knows their place

3b. Anticipating Positive Outcomes of Integrating the HNP into this Clinic.

Comments were coded in this category if they indicated that there have been positive outcomes through benefits to patients, staff, and the HNPs. Examples of comments coded in this category include: 
- The introduction of this type of cadre has contributed to regular follow-up of patients and early commencement of treatment.

- Integrating HIV Nurse Practitioner into our health care facility should in fact promote a holistic and comprehensive care, treatment and support.

\section{3c. Concerns with Integrating the HNP into this Clinic.}

Comments were coded in this category if they indicated concerns regarding the role, acceptance of the new role, adverse impacts on the operation of the clinic, the patients, the staff or patient wards, the need for strategies to ensure high quality of care and overloading the HNPs with work and not adequately rewarding them financially. Examples of comments coded in this category include:

- Allowing HIV nurse practitioners fully independently without regular supervision may compromise quality of care

- Normally they miss the basics that is, in the physical examination, not done thoroughly and also taking the vitals

- If current staff structure are not well oriented to the HIV nurse practitioner roles - this may cause the nurse to be isolated and not supported by other clinicians

- As more get trained more staff will be pulled away from general nursing duties i.e. we need more nurses

- All systems need feedback but a weekly cumulative report on statistics and services provided will guarantee this

- The major concern is work burden/overload on this nurse, may we consider to motivate them

Question 4: Supervisors' Concerns or Perception of Potential Problems in Integrating the HNP into the Zambian Health System.

Three codes emerged from responses asking supervisors about their concerns or perceptions of potential problems in integrating the HNP into the Zambian health system: (4a) anticipating no problems with integration of the HNP into the Zambian health system, (4b) anticipating positive outcomes of integrating the HNP into the Zambian health system and (4c) concerns with integrating the HNP into the Zambian health system.

4a. Anticipating No Problems with Integrating of the HNP into the Zambian Health System.

Comments were coded in this category if they indicated that there have been no problems, and/or anticipation of no problems for staff or patients with integration of the HNP into the Zambian health system. Examples of comments coded in this category include:

- There should be no problems because nurse practitioners are an acceptable cadre throughout the world, therefore there should be no obstacles in getting licensing and establishments updated

- I have no concerns or potential problems integrating HIV nurse practitioners into the Zambian health system, they are a better substitute to the shortage of clinicians

- There are no potential problems; it is a welcome idea

- No major concerns if consultation and referral systems are strengthened

- So far no problems noted as the introduction of this cadre has greatly contributed to good service delivery to ART clients

4b. Anticipating Positive Outcomes of Integrating the HNP into the Zambian Health System.

Comments were coded to this category if they indicated that there have been positive outcomes to patients, staff and the HNPs. Examples of comments coded in this category include:

- Instead it's a step ahead as there are many patients waiting to be seen by one clinician, it will really be a breakthrough in the Zambian health system

- There are few clinicians in the Zambian health system, integration of the HIV nurse practitioner will increase number of medical practitioners

- So far no problems noted as the introduction of this cadre has greatly contributed to good services deliver to ART clients

\section{4c. Concerns with Integrating the HNP into the Zambian Health System.}

Comments were coded in this category if they indicated concern for negatively impacting the nursing shortage, overuse of nurses, HNP knowledge of the need for referral and consultation, HNP supervision, ongoing HNP education, diploma recognition, or financial incentives to motivate HNPs. Examples of comments in this category include:

- Nurse practitioners might not be recognized by the Zambian health system and they might not be ready to give the practitioner and incentive, failure to sustain the nurse practitioner program by the Zambian health system

- Supervision of the nurse practitioners should be ongoing to avoid unnecessary problems

- Need continuous medical education, may need continuous updates on new interventions and strategies

ISSN 1925-4040 E-ISSN 1925-4059 
- We must clarify from the beginning each one's role, the job description, and responsibilities

- Shortage will be created in departments where these nurses (those trained as HNP) are coming from

\section{Question 5: Supervisors' Suggestions About Strategies to Improve the HNP Program.}

Five codes emerged from responses asking supervisors to share comments that will improve the program: (5a) clarify HNP role and scope of practice, (5b) need for recognizing, compensating, or motivating HNPs, (5c) supportive of the program and the HNP role, (5d) suggestions for selection of participants, program design, training, and ongoing HNP education strategies and (5e) general concerns, potential problems.

\section{5a. Clarify HNP Role and Scope of Practice.}

Comments were coded in this category if they indicated the need to clarify the HNP role, responsibilities, and scope of practice. Examples of comments in this category include:

- A clear distinction of what complications can be managed and which to refer

- There might be confusion on whether the HIV nurse practitioner is a new cadre that should only concentrate on the new skills or can still be used as a nurse in case of shortages

5b. Need for Recognizing, Compensating, or Motivating HNPs.

Comments were coded in this category if they indicated the need for recognition, compensation or motivation for the HNPs. Examples of comments in this category include:

- I don't see any problem except the financial impact it may have from the demand for recognition of this diploma and mentoring reward

- HNPs need to be motivated compensated

\section{5c. Supportive of the Program and the HNP Role.}

Comments were coded in this category if they indicated support for the program design, curriculum, or integration of the HNP role into Zambian health care. Examples of comments in this category include:

- So far the two nurses I have worked with are doing a great job, the program should continue with empowering them with more knowledge

- Where these nurses work there are less problems in the coordination of the ART program

5d. Suggestions for Selection of Participants, Program Design, Training, and Ongoing HNP Education Strategies.

Published by Sciedu Press
Comments were coded in this category if they indicated suggestions to improve the program by selection of program participants, the program design, training, or curriculum content, and ways to improve the HNP resources after the program. Examples of comments in this category include:

- Priority should be given to NPs serving in rural areas

- Priority should be given to rural health staff where they have one or few clinicians

- Mentees need other references apart from the mentor (books, internet)

- Train NP for leadership of rural health centre

- Additionally, let them also undergo clinical teaching skills course

- They should also be trained as mentors for ART

- Nurses will need refresher courses so that they are always updated with new coming literature on the care and treatment of HIV/AIDS

\section{5e. General concerns, potential problems.}

Comments were coded in this category if they indicated potential problems or areas to improve including quality of care, scope of practice, and regulatory issues. Examples of comments in this category include:

- Allowing HIV nurse practitioners fully independently without regular supervision may compromise quality of care

- HNP not backed by law

\section{Discussion}

The findings from this study support findings from previous research that demonstrate positive impacts of task-shifting of HIV services from physicians to nurses, although there are several limitations that must be acknowledged. Only $65 \%$ of the supervisors of the 92 graduates returned the surveys. Although the project's Monitoring and Evaluation Team members suggested that graduates should deliver the surveys to their supervisors (instead of having project staff mail the surveys), the response rate may have been higher if the surveys had been mailed. A second limitation is the failure to collect demographic data about the supervisors who responded to the anonymous surveys. These data could have been useful in identifying whether there were differences in perceptions about the program based on location of the clinic, or other demographic characteristics of the respondents. A final limitation was that the instrument used in the study was developed by the researchers, and although it had face validity, there was no assessment of its reliability.

Despite these limitations, the study findings contribute to the emerging body of research to assess the impact of taskshifting programs in low resource countries like Zambia. 
The supervisors who responded to the surveys in this study indicated that they understood the role for which the students were being prepared to include collaborating with other clinicians, providing comprehensive care to HIV and AIDS patients and their families, providing care with the clinical officer or physician is not present, screening, testing, enrollment, counseling, follow-up, outreach, and coordination of services for HIV/ART clients. The supervisors also identified many positive aspects of the task-shifting program including improving access to care, managing OIs, caring for stable patients, initiating ART, and freeing the physician to manage more complex cases. These findings are consistent with findings reported in our literature review.

Although the supervisors identified many positive aspects of the HNP program, the concerns that they identified have significant implications for ongoing efforts to improve the program and to integrate the graduates into the Zambian health system. One respondent noted concern about negative impact on health outcomes if the HNPs do not receive adequate support and supervision, leading to failure to refer patients who may need physician management. Other concerns that were identified included (a) problems with retaining the HNP graduates if the health system does not recognize the role and identify incentives and to reflect their additional education and job responsibilities; (b) the need to develop a system to monitor and ensure quality of care of the HNP graduates; (c) exacerbating the shortage of nurses in other areas of the health system if the HNP graduates are pulled to serve only HIV patients; and (d) the potential for excessive workload and burnout of the graduates due to the heavy demand for their services.

These findings suggest that it will be important to develop clear roles and guidelines for the HNP role, ensure ongoing supervision and support, monitor the quality of care provided, and ensure an adequate distribution of nurses across all components of the Zambian health system. As HIV services become increasingly focused on providing log-term chronic disease management, it will be important to assess whether the HNP program should prepare nurses for more general advanced practice roles, rather than focus specifically on HIV.

\section{Conclusion}

Findings from this study suggest that supervisors generally had positive perceptions of the potential impact of the HNP role on the Zambian health system, consistent with findings from other studies of task-shifting to increase access to care for patients with HIV. However, there were concerns identified related to the need for clarifying the role, providing adequate incentives and ongoing support, and for monitor- ing the impact of the program on patient outcomes and on nursing workforce needs in Zambia. These findings have implications for ongoing monitoring, evaluation, and revision of programs aimed at task-shifting to improve access to care in resource-constrained settings.

\section{ACKNOWLEDGEMENTS}

The Zambian HIV Nurse Practitioner Program was originally developed and coordinated under the leadership of the General Nursing Council of Zambia (GNC). The GNC expresses sincere thanks to the Sparkman Center for Global Health at the University of Alabama at Birmingham (UAB), USA; the United States Centers for Disease Control and Prevention (CDC), Zambia Office; AIDSRelief; the Centre for Infectious Diseases Research in Zambia (CIDRZ); the Zambia Prevention Care and Treatment (ZPCT II); the University of Zambia; and the Zambia Ministry of Health for financial or technical support for the development of this curriculum and teaching and learning materials. The GNC also gratefully acknowledges the significant contributions from all the members of the HIV Nurse Practitioner Curriculum Committee and the support of the institutions listed below towards the development of this curriculum: Catholic Medical Mission Board; HSSP/JHPIEGO; International Training \& Education Center on HIV (ITECH); Lusaka Schools of Nursing and Midwifery; PAHO/WHO Collaborating Center for International Nursing, UAB School of Nursing; University of Maryland, USA; University Teaching Hospital; Zambia Union of Nurses Organisation; World Health Organization; and Zambia Prevention Care and Treatment (ZPCT II).

The authors of this manuscript also wish to offer special thanks to Prudencia Mweemba, RN, MSN, PhD, Formerly the Head of the Department of Nursing Sciences, University of Zambia School of Medicine (Deceased in June 2014), for her commitment to this program and many others that have positively impacted nursing care in Zambia. Special thanks also to Moses Sinkala M.D., Formerly the Medical Director of the Zambia HIV Nurse Practitioner Program, and Medical Director of the Catholic Medical Missions Board Zambia Office (Deceased in December 2012), for his unwavering support of efforts to improve health care for Zambians. The authors also wish to recognize and thank Lloyd Mulenga, M.D., University of Zambia School of Medicine, and current Medical Director of the Zambia HIV Nurse Practitioner Program for his ongoing support and direction of the HNP program and for his continued commitment to the success of the program.

\section{CONFLiCtS OF INTEREST Disclosure}

The authors declare that they have no competing interests. 


\section{REFERENCES}

[1] Naicker S, Eastwood JB, Tutt RC. Shortage of healthcare workers in sub-Saharan Africa: a neurological perspective. Clin Neph. 2010; 74(Supplement 1): 129-33.

[2] Campbell J, Dussault G, Buchan J, et al. A universal truth: no health without a workforce. Recife, Brazil: Geneva, Global Health Workforce Alliance and World Health Organization. 2013.

[3] Office of the United States Global AIDS Coordinator. The President's Emergency Plan for AIDS Relief Report on Work Force Capacity and HIV/AIDS. 2006

[4] United Nations. Transforming our world: The 2030 agenda for sustainable development. 2015

[5] Central Intelligence Agency. The World Factbook Library: Zambia - People and Society 2014 [updated June 20, 2014]. Available from: https://www.cia.gov/library/publications/the-w orld-factbook/geos/za.html

[6] United Nations Office of the High Representative for the Least Developed Countries Landlocked Developing Countries and Small Island Developing States (UN-OHRLLS). State of the least developed countries 2014. New York, NY: United Nations, 2014.

[7] United Nations Children's Fund (UNICEF). Zambia HIV and AIDS Factsheet 2010 [January 21, 2014]. Available from: http://www. unicef.org/zambia/5109_8459.html

[8] Central Intelligence Agency. The World Factbook Library: Zambia, Country Comparison: HIV/AIDS-Adult Prevalence Rate 2012 [January 29, 2015]. Available from: https://www.cia.gov/library/publications/the-w orld-factbook/rankorder/2155rank.html

[9] Center for Global Health Centers for Disease Control and Prevention. CDC in Zambia Factsheet. Atlanta, GA: 2013.

[10] Dzekedzeke K, Siziya S, Fylkesnes K. The impact of HIV infection on adult mortality in some communities in Zambia: a cohort study. Tropical Medicine and International Health. 2008; 13(2): 152-61. PMid:18304260. http://dx.doi.org/10.1111/j.1365-3156. $2007.01985 . \mathrm{x}$

[11] United Nations Inter-agency Group for Child Mortality Estimation. Levels \& Trends in Child Mortality. 2014.

[12] World Health Organization. Child mortality: The partnership for maternal, newborn \& child health. 2011

[13] Republic of Zambia Ministry of Health. Human Resources for Health Strategic Plan (Draft) 2006-2010. 2005.

[14] Republic of Zambia Ministry of Health. National Human Resources for Health Strategic Plan 2011-2015. Lusaka, Zambia: Ministry of Health, 2011.

[15] General Nursing Council of the Republic of Zambia. The Nurses and Midwives Act No. 31 of 1997 [cited 2015 2/21/15]. Available from: http://www.gnc.org. $\mathrm{zm} /$ index . php?option=com content\&view=article\&id=122\&Itemid=105

[16] Msidi E, Sinkala M, Bositis A, et al. The Zambian HIV nurse practitioner diploma program: preliminary outcomes from first cohort of Zambian nurses. International Journal of Nursing Education Scholarship. $2011 ; 8(1)$

[17] Mulenga UH, Chanda D, Gashongore I, et al. Follow-up evaluation of first two cohorts of graduates of the Zambian HIV nurse practitioner program. Intl J of Afr Nur Sci. 2015; (2): 1-9. http: //dx.doi.org/10.1016/j.ijans.2015.01.002

[18] World Health Organization. Task Shifting: Rational Redistribution of Tasks Among Health Workforce Teams: Global Recommendations and Guidelines. Geneva, Switzerland: World Health Organization, 2008 Contract No.: ISBN 9789241596312.

[19] Green AV, Patten G, Davies M, et al. Clinical mentorship of nurse initiated antiretroviral therapy in Khayelitsha, South Africa: a quality of care assessment. PLoS ONE. 2014; 9(6). http://dx.doi.org /10.1371/journal.pone.0098389

[20] Barton G, Farial L, Bachmann M, et al. Cost-effectiveness of nurseled versus doctor-led antiretroviral treatment in South Africa: pragmatic cluster randomised trial. Tropical Medicine and International Health. 2013; 18(6): 769-77. http://dx.doi.org/10.111/tmi 12093

[21] Sanne I, Orrell C, Fox MP, et al. Nurse versus doctor management of HIV-infected patients receiving antiretroviral therapy (CIPRASA): a randomized non-inferiority trial. The Lancet. 2010 June 16. http://dx .doi .org/10.1016/50140-6736(10)60894-x

[22] Callaghan M, Ford N, Scheider H. A systematic review of taskshifting for HIV treatment and care in Africa. Human Resources for Health. 2010; 8(8).

[23] Emdin C, Chong N, Millson P. Non-physician clinician provided HIV treatment results in equivalent outcomes as physician-provided care: a meta-analysis. J of the Int AIDS Soc. 2013; 16(18445).

[24] Iwu E, Holzemer W. Task shifting of HIV management from doctors to nurses in Africa: Clinical outcomes and evidence on nurse self-efficacy and job satisfaction. AIDS Care. 2014; 26(1): 42-52. http://dx.doi.org/10.1080/09540121.2013.793278

[25] Kredo T, Adeniyi F, Batenganya M, et al. Task shifting from doctors to non-doctors for initiation and maintenance of antiretriviral therapy. Cochrane Database of Systematic Reviews. 2014; (7) http://dx.doi.org/10.1002/14651858.CD007331.pub3

[26] Bemelmans M, Van Den Akker T, Ford N, et al. Providing universal access to antiretroviral therapy in Thyolo, Malawi through task shifting and decentralization of HIV/AIDS care. Tropical Medicine and International Health. 2010; 15(12): 1413-20. http://dx.doi.org /10.1111/j.1365.2010.02649.x

[27] Boulle C, Kouanfack C, Laborde-Balen G, et al. Task shifting HIV care in rural district hospitals in Cameroon: evidence of comparible antiretroviral treatment-related outcomes between nurses and physicans in the Stratall ANRS/ESTER trial. J Acq Imm Def Syn. 2013; $62(5)$.

[28] Grimsrud A, Kaplan R, Bekker L, et al. Outcomes of a nursemanaged service for stable HIV-positive patients in a large South African public sector antiretroviral therapy programme. Tropical Medicine and International Health. 2014; 19(9): 1029-39.

[29] Dambisya Y, Matinhure S. Policy and programmatic implications of task-shifting in Uganda: A case study. BMC Health Services Research. 2012; 12(61).

[30] Morris MB, Tambatamba-Chapula B, Chi BH, et al. Use of taskshifting to rapidly scale-up HIV treatment services: experiences from Lusaka, Zambia. BMC Health Services Research. 2009; 9(5): 21. PMid:19183496

[31] O’Malley G, Asrat L, Sharma A, et al. Nurse task shifting for antiretroviral treatment services in Namibia: Implementation research to move evidence into action. PLoS ONE. 2014; 9(3): e92014. http://dx.doi.org/10.1371/journal.pone.0092014

[32] Cohen R, Lynch S, Bygrave H, et al. Antiretroviral treatment outcomes from a nurse-driven, community-supported HIV/AIDS treatment programme in rural Lesotho: observational cohort assessment at two years. Journal of the International AIDS Society. 2009; 12(23) http://dx.doi.org/10.1186/1758-2652-12-23

[33] Mdege N, Chindove S, Ali S. The effectiveness and cost implications of task-shifting in the delivery of antiretroviral therapy to HIVinfected patients: a systematic review. Health Policy and Planning 2013; 28: 223-36. http://dx.doi.org/10.1093/heapol/czs0 58

[34] Asfaw E, Dominis S, Palen J, et al. Patient satisfaction with task shifting of antiretroviral services in Ethiopia: implications for univer- 
sal health coverage. Health Policy and Planning. 2014; 29(ii): 50-8. [35] Foster N, McIntyre D. Economic evaluation of task-shifting aphttp://dx.doi.org/10.1093/heapol/czu072 proaches to the dispensing of anti-retroviral therapy. Human Resources for Health. 2012; 10(32). 